\title{
RANK PROPERTIES IN FINITE INVERSE SEMIGROUPS
}

\author{
MARIA ISABEL MARQUES RIBEIRO \\ Departamento de Matemática, Universidade dos Açores, Rua da Mãe de Deus, \\ 9500 Ponta Delgada (Açores), Portugal (isamr@uac.pt)
}

(Received 24 November 1998)

\begin{abstract}
Two possible concepts of rank in inverse semigroup theory, the intermediate $I$-rank and the upper $I$-rank, are investigated for the finite aperiodic Brandt semigroup. The so-called large $I$-rank is found for an arbitrary finite Brandt semigroup, and the result is used to obtain the large rank of the inverse semigroup of all proper subpermutations of a finite set.
\end{abstract}

Keywords: inverse semigroup; Brandt semigroup; rank; intermediate $I$-rank; upper $I$-rank; large $I$-rank AMS 1991 Mathematics subject classification: Primary 20M10

\section{Introduction}

In previous papers $[3,4]$, Howie and I investigated various concepts of rank applied to certain known semigroups. One of the semigroups involved was the finite aperiodic Brandt semigroup $B_{n}$, whose definition is recalled below. This is of course an inverse semigroup, and if we choose to regard it in this way the questions regarding rank change significantly. This is because the inverse subsemigroup generated by a subset $A$ of an inverse semigroup $S$ will usually be larger than the subsemigroup generated by $A$. To avoid confusion we shall denote the inverse subsemigroup by $\langle\langle A\rangle$ and the subsemigroup by $\langle A\rangle$, and we shall say that a subset $A$ of an inverse semigroup $S$ is $I$-independent if, for all $a$ in $A$,

$$
a \notin\langle\langle A \backslash\{a\}\rangle\rangle
$$

Applying ideas due to Marczewski [5], we can consider the following rank functions on a finite inverse semigroup $S$ :

(i) $r_{1}^{I}(S)=\max \{k$ : every subset $U$ of $S$ of cardinality $k$ is $I$-independent $\}$;

(ii) $r_{2}^{I}(S)=\min \{k$ : there exists a subset $U$ of $S$ of cardinality $k$ such that $\langle\langle U\rangle\rangle=S\}$;

(iii) $r_{3}^{I}(S)=\max \{k$ : there exists a subset $U$ of $S$ of cardinality $k$ that is $I$-independent and such that $\langle\langle U\rangle\rangle=S\}$;

(iv) $r_{4}^{I}(S)=\max \{k$ : there exists a subset $U$ of $S$ of cardinality $k$ that is $I$-independent $\}$; 
(v) $r_{5}^{I}(S)=\min \{k$ : every subset $U$ of $S$ of cardinality $k$ has the property that $\langle\langle U\rangle\rangle=S\}$.

As in the semigroup case we can easily show that

$$
r_{1}^{I}(S) \leqslant r_{2}^{I}(S) \leqslant r_{3}^{I}(S) \leqslant r_{4}^{I}(S) \leqslant r_{5}^{I}(S),
$$

and for convenience we shall use the following terminology:

$r_{1}^{I}(S)$ : the small I-rank;

$r_{2}^{I}(S):$ the lower I-rank;

$r_{3}^{I}(S)$ : the intermediate I-rank;

$r_{4}^{I}(S):$ the upper $I$-rank; and

$r_{5}^{I}(S)$ : the large I-rank.

Of the five ranks the most interesting are $r_{2}^{I}, r_{3}^{I}$ and $r_{4}^{I}$, all of which coincide with the dimension when $S$ is a vector space. The least interesting is $r_{1}^{I}$ : for an inverse semigroup $S$ it is easy to see that $r_{1}^{I}(S)=1$ unless $S$ is a semi-lattice $E$, and $r_{1}^{I}(E)=2$ unless $E$ is a chain, and $r_{1}^{I}(E)=|E|$.

From $[\mathbf{3}, \mathbf{4}]$ we know that the corresponding semigroup ranks $r_{1}, \ldots, r_{5}$, when applied to $B_{n}$, give (for $n \geqslant 2$ )

$$
\begin{gathered}
r_{1}\left(B_{n}\right)=1, \quad r_{2}\left(B_{n}\right)=n, \quad r_{3}\left(B_{n}\right)=2 n-2, \\
r_{4}\left(B_{n}\right)=\left\lfloor n^{2} / 4\right\rfloor+n, \quad r_{5}\left(B_{n}\right)=n^{2}-n+3 .
\end{gathered}
$$

As remarked above, $r_{1}^{I}\left(B_{n}\right)=1$, and, from [1], we have that $r_{2}^{I}\left(B_{n}\right)=n-1$. In this note we investigate $r_{3}^{I}\left(B_{n}\right), r_{4}^{I}\left(B_{n}\right)$ and $r_{5}^{I}\left(B_{n}\right)$.

For unexplained terms in semigroup theory see [2].

\section{The intermediate $I$-rank}

Recall that $B_{n}=(\{1,2, \ldots, n\} \times\{1,2, \ldots, n\}) \cup\{0\}$, and that

$$
\begin{aligned}
(i, j)(k, l) & = \begin{cases}(i, l), & \text { if } j=k, \\
0, & \text { otherwise, }\end{cases} \\
(i, j) 0 & =0(i, j)=00=0 .
\end{aligned}
$$

Theorem 2.1. With the above definitions, and with $n \geqslant 2$,

$$
r_{3}^{I}\left(B_{n}\right)=n-1 .
$$

Proof. It is easy to see that $r_{3}^{I}\left(B_{n}\right) \geqslant n-1$, for the subset

$$
A=\{(1,2),(1,3), \ldots,(1, n)\}
$$


is $I$-independent. To see that $\langle\langle A\rangle\rangle=B_{n}$, notice first that $0 \in\langle\langle A\rangle\rangle$, that $(1,1)=$ $(1,2)(1,2)^{-1} \in\left\langle\langle A\rangle\right.$, and that $(i, 1)=(1, i)^{-1} \in\langle\langle A\rangle\rangle$ for all $i \in\{1,2, \ldots, n\}$. Then, finally, $(i, j)=(i, 1)(1, j) \in\langle\langle A\rangle\rangle$ for all $i, j \in\{1,2, \ldots, n\}$.

To show that no larger $I$-independent generating set can be found, we use a graphical technique. To each subset $A$ of $B_{n} \backslash\{0\}$ we associate an (undirected) graph $\Gamma(A)$ whose vertices are labelled $1,2, \ldots, n$, and where there is an edge, $i \longleftrightarrow j$, between $i$ and $j$ if and only if $(i, j) \in A$.

Within a graph $\Gamma$ we shall say that a sequence of vertices

$$
\left(v_{0}, v_{1}, \ldots, v_{m-1}, v_{m}\right)
$$

is a path between $v_{0}$ and $v_{m}$ if $v_{i} \longleftrightarrow v_{i+1}$ is an edge in $\Gamma$ for $i=0,1, \ldots, m-1$. It is a proper path if $m \geqslant 2$ and if $v_{1}, \ldots, v_{m-1}$ are all distinct from $v_{0}$ and $v_{m}$.

It is easy to see that $\langle\langle A\rangle\rangle=B_{n}$ if and only if the graph $\Gamma(A)$ is connected, that is, if and only if there is a path in $\Gamma(A)$ linking any two vertices. Also, $A$ is $I$-independent if and only if the graph $\Gamma(A)$ is $I$-independent, in the sense that, for all $i, j$ in $\{1,2, \ldots, n\}$, there cannot exist both an edge and a proper path between $i$ and $j$.

We therefore require to prove that, for any connected $I$-independent graph $\Gamma$ with no repeated edges,

$$
|V(\Gamma)|=n \Longrightarrow|E(\Gamma)| \leqslant n-1 \text {. }
$$

We do this by induction on $n$, it being clear by direct verification that a graph with two vertices and at least two edges cannot be both $I$-independent and connected: if there are just two edges, the possibilities are

$$
\{(1,1),(1,2)\}, \quad\{(1,1),(2,2)\}, \quad\{(1,2),(2,2)\},
$$

and of these the first and the third are not $I$-independent, and the second is not connected; if there are three edges the only possibility is

$$
\{(1,1),(2,2),(1,2)\}
$$

and this set is not $I$-independent.

Let $\Gamma$ be both connected and $I$-independent, and suppose that $|V(\Gamma)|=n$. Notice first that $\Gamma$ cannot contain any loops $x \longleftrightarrow x$, since there is an edge $x \longleftrightarrow y$ for some $y \neq x$, and then we would have both an edge $x \longleftrightarrow x$ and a proper path $(x, y, x)$. Choose a vertex $x$ at random, and suppose that there are edges $x \longleftrightarrow y_{j}(j=1,2, \ldots, m)$. Notice that there cannot be any edges $y_{j} \longleftrightarrow y_{k}(j, k \in\{1, \ldots, m\})$, because any such edge would give rise to a proper path

$$
\left(x, y_{j}, y_{k}\right)
$$

We form a new graph $\Gamma^{\prime}$ whose set of vertices is $V(\Gamma) \backslash\{x\}$, and whose edges are those of $\Gamma$, except that all the edges $x \longleftrightarrow y_{j}(j=2, \ldots, m)$ are replaced by $y_{1} \longleftrightarrow y_{j}$. So, 
for example, if $n=6$ and $\Gamma$ is

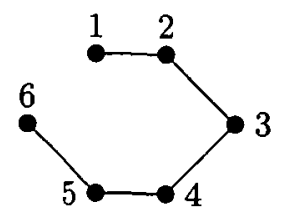

then, taking $x=3$ and $y_{1}=2$, we see that $\Gamma^{\prime}$ is

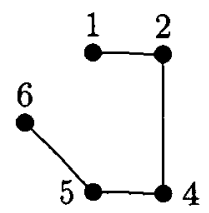

We show first that $\Gamma^{\prime}$ is connected. Certainly, any two vertices $i$ and $j$ of $\Gamma^{\prime}$ are linked by a path in $\Gamma$. If this path does not pass through $x$, then it is a path in $\Gamma^{\prime}$, and there is nothing to prove. If the path

$$
(i, \ldots, z, x, t, \ldots, j)
$$

passes through $x$ (and we may assume that the path is without loops, so that this happens only once) then $z, t \in\left\{y_{1}, \ldots, y_{m}\right\}$. If $z \neq y_{1}$ and $t \neq y_{1}$, then $\left(i, \ldots, z, y_{1}, t, \ldots, j\right)$ is a path in $\Gamma^{\prime}$. If $z=y_{1}$, then $t \neq y_{1}$, and $\left(i, \ldots, y_{1}, t, \ldots, j\right)$ is a path in $\Gamma^{\prime}$. If $t=y_{1}$, then $z \neq y_{1}$, and $\left(i, \ldots, z, y_{1}, \ldots, j\right)$ is a path in $\Gamma^{\prime}$. Thus $\Gamma^{\prime}$ is connected.

To show that $\Gamma^{\prime}$ is $I$-independent, suppose that $i \longleftrightarrow j$ is an edge in $\Gamma^{\prime}$ and that there is also a proper path $P:(i, \ldots, j)$ in $\Gamma^{\prime}$. If $P$ does not pass through $y_{1}$, then it is in fact a proper path in $\Gamma$, and we have a contradiction. Suppose, therefore, that $P$ passes through $y_{1}$,

$$
\left(i, \ldots, u, y_{1}, v, \ldots, j\right)
$$

where at least one of the edges $u \longleftrightarrow y_{1}, y_{1} \longleftrightarrow v$ is absent from $\Gamma$. From the construction of $\Gamma^{\prime}$ it follows that if $u \longleftrightarrow y_{1}$ is absent, then $u \longleftrightarrow x$ is an edge of $\Gamma$; and, similarly, if $y_{1} \longleftrightarrow v$ is absent, then $x \longleftrightarrow v$ is an edge in $\Gamma$. Hence, we have one of the following proper paths in $\Gamma$ :

$$
\left(i, \ldots, u, x, y_{1}, v, \ldots, j\right), \quad\left(i, \ldots, u, y_{1}, x, v, \ldots, j\right), \quad(i, \ldots, u, x, v, \ldots, j) .
$$

By induction we may assume that $\left|E\left(\Gamma^{\prime}\right)\right| \leqslant n-2$. Hence,

$$
|E(\Gamma)|=\left|E\left(\Gamma^{\prime}\right)\right|+1 \leqslant n-1 .
$$

This completes the proof.

\section{The upper I-rank}

In fact it is fairly easy to modify the proof of Theorem 2.1 to also deal with the upper $I$-rank $r_{4}^{I}\left(B_{n}\right)$. 
Theorem 3.1. Let $n \geqslant 2$. Then $r_{4}^{I}\left(B_{n}\right)=n$.

Proof. It is easy to see that $r_{4}^{I}\left(B_{n}\right) \geqslant n$, for the subset

$$
A=\{(1,1),(2,2), \ldots,(n, n)\}
$$

is $I$-independent.

To show that no larger $I$-independent set can be found, we use the same graphical technique, and now we need consider only the $I$-independent property of the graph.

We require to prove that, for any $I$-independent graph $\Gamma$ with no repeated edges,

$$
|V(\Gamma)|=n \Longrightarrow|E(\Gamma)| \leqslant n .
$$

We do this by induction on $n$, it being clear by direct verification that no graph with two vertices and three or more edges can be $I$-independent: there cannot in fact be more than three edges, and if there are just three edges the only possibility is

$$
\{(1,1),(1,2),(2,2)\},
$$

which is certainly not $I$-independent.

Let $\Gamma$ be $I$-independent, and suppose that $|V(\Gamma)|=n$. Choose a vertex $x$ at random, and form a new graph $\Gamma^{*}$ such that $V\left(\Gamma^{*}\right)=V(\Gamma) \backslash\{x\}$ and whose edges are all the edges of $\Gamma$ except $x \longleftrightarrow x$ if the loop $x \longleftrightarrow x$ is present, all the edges of $\Gamma$ if the loop $x \longleftrightarrow x$ is not present and if there does not exist $y$, with $y \neq x$, such that $x \longleftrightarrow y$ is an edge in $\Gamma$ and, if there are edges $x \longleftrightarrow y_{j}(j=1,2, \ldots, m), \Gamma^{*}$ has the same set of edges of $\Gamma$ except that all the edges $x \longleftrightarrow y_{j}(j=2, \ldots, m)$ are replaced by $y_{1} \longleftrightarrow y_{j}$. Suppose first that the loop $x \longleftrightarrow x$ is present. Then there can be no edges $x \longleftrightarrow y$ with $y \neq x$, for otherwise we have a proper path $(x, y, x)$. In this case, $E\left(\Gamma^{*}\right)$ consists of all the edges of $E(\Gamma)$ except $x \longleftrightarrow x$, and $\Gamma^{*}$ is certainly independent. By induction, since $\left|V\left(\Gamma^{*}\right)\right|=n-1$, we must have $\left|E\left(\Gamma^{*}\right)\right| \leqslant n-1$, and so it follows that

$$
|E(\Gamma)|=\left|E\left(\Gamma^{*}\right)\right|+1 \leqslant n,
$$

as required.

Suppose now that the loop $x \longleftrightarrow x$ is not present. If there does not exist $y$ such that $x \longleftrightarrow y$ is an edge in $\Gamma$, then $\Gamma^{*}$ is certainly independent, and $|E(\Gamma)|=\left|E\left(\Gamma^{*}\right)\right| \leqslant n-1$ by the induction hypothesis. So suppose that there are edges $x \longleftrightarrow y_{j}(j=1,2, \ldots, m)$. Notice there cannot be any edges $y_{j} \longleftrightarrow y_{k}(j, k \in\{1, \ldots, m\})$, because any such edge would give rise to a proper path

$$
\left(x, y_{j}, y_{k}\right)
$$

Now, let $\Gamma^{*}$ have the same set of edges as $\Gamma$, except that all the edges $x \longleftrightarrow y_{j}(j=$ $2, \ldots, m)$ are replaced by $y_{1} \longleftrightarrow y_{j}$. The diagrams (2.1) and (2.2) are as relevant here as in the earlier proof. Indeed, the proof that $\Gamma^{*}$ is $I$-independent is in effect identical to the proof in the last section that $\Gamma^{\prime}$ is $I$-independent, and is omitted.

By induction we may assume that $\left|E\left(\Gamma^{*}\right)\right| \leqslant n-1$. Hence,

$$
|E(\Gamma)|=\left|E\left(\Gamma^{*}\right)\right|+1 \leqslant n .
$$

This completes the proof. 


\section{The large $I$-rank}

We now investigate $r_{5}^{I}\left(B_{n}\right)$. In fact, we consider something more general, and examine the finite Brandt semigroup $B(G, n)$ for an arbitrary finite group $G$. Recall that

$$
B(G, n)=(\{1,2, \ldots, n\} \times G \times\{1,2, \ldots, n\}) \cup\{0\},
$$

and that the multiplication is given by

$$
\begin{aligned}
(i, a, j)(k, b, l) & = \begin{cases}(i, a b, l), & \text { if } j=k, \\
0, & \text { otherwise, }\end{cases} \\
0(i, a, j) & =(i, a, j) 0=00=0 .
\end{aligned}
$$

By analogy with [4, Theorem 3], we have the following theorem.

Theorem 4.1. Let $S$ be a finite inverse semigroup, and let $V$ be the largest proper inverse subsemigroup of $S$. Then $r_{5}^{I}(S)=|V|+1$.

We now have the following theorem.

Theorem 4.2. Let $n \geqslant 2$. Then $r_{5}^{I} B(G, n)=\left(n^{2}-2 n+2\right)|G|+2$.

Proof. We begin by describing all of the inverse subsemigroups of $S=B(G, n)$. Let $V$ be an inverse subsemigroup of $S$. Denote $\{1, \ldots, n\}$ by $[n]$.

Define a relation $\rho_{V}$ on $[n]$ by

$$
(i, j) \in \rho_{V} \Leftrightarrow(\exists g \in G)(i, g, j) \in V .
$$

It is easy to see that the relation $\rho_{V}$ is symmetric and transitive, but, in general it is not reflexive. Let $M=\left\{i \in[n]:(i, i) \in \rho_{V}\right\}$.

In fact, $V \backslash 0 \subseteq M \times G \times M$. For suppose that $i \notin M$; then there cannot exist $j$ such that $(i, j) \in \rho_{V}$, since then we would have $(j, i) \in \rho_{V}$ by symmetry and so $(i, i) \in \rho_{V}$ by transitivity. Now, the relation $\bar{\rho}_{V}=\rho_{V} \cap(M \times M)$ is an equivalence on $M$. Let $C \subseteq M$ be a $\bar{\rho}_{V}$-class. For all $i, j \in C$, let $H_{i, j}$ be the (non-empty) subset of $G$ defined by $H_{i, j}=\{g \in G:(i, g, j) \in V\}$. For all $i, j, k \in C$ we have $H_{i, j} H_{j, k} \subseteq H_{i, k}$, and, in particular, $H_{i, i}^{2} \subseteq H_{i, i}$ for all $i \in C$.

Let $i, j, k, l \in C$ and let $p \in H_{i, k}, q \in H_{l, j}$. Then $p^{-1} \in H_{k, i}, q^{-1} \in H_{j, l}$ and $p H_{k, l} q \subseteq$ $H_{i, j}, p^{-1} H_{i, j} q^{-1} \subseteq H_{k, l}$.

Hence,

$$
\left|H_{k, l}\right|=\left|p H_{k, \iota} q\right| \leqslant\left|H_{i, j}\right|=\left|p^{-1} H_{i, j} q^{-1}\right| \leqslant\left|H_{k, l}\right|,
$$

and, for all $i, j, k, l \in C$, we have $\left|H_{k, l}\right|=\left|H_{i, j}\right|$.

Now we choose and fix $i$ in $C$ and denote the group $H_{i, i}$ by $H_{V}^{C}$, and choose for each $j \in C$ an element $p_{j}$ in $H_{i, j}$, arbitrarily.

Then, every $H_{j, k}$ with $j, k \in C$ is the double coset $p_{j}^{-1} H_{V}^{C} p_{k}$, and

$$
V=\{0\} \cup \bigcup_{C \in M / \bar{\rho}_{V}} \bigcup_{j, k \in C}\{j\} \times p_{j}^{-1} H_{V}^{C} p_{k} \times\{k\} .
$$


The choice of $i$ and of the elements $p_{j}$ is arbitrary, but $p_{j}^{-1} H_{V}^{C} p_{k}$ will always coincide with $H_{j, k}$.

Conversely, choose a subset $M$ of $[n]$, and let $\rho$ be an equivalence on $M$. For each $\rho$-class $C$, choose and fix an element $i$ in $C$ and let $H^{C}$ be a subgroup of $G$. For each $j$ in $C$ choose $p_{j}$ in $G$ arbitrarily. Then

$$
V=\{0\} \cup \bigcup_{C \in M / \rho} \bigcup_{j, k \in C}\{j\} \times p_{j}^{-1} H^{C} p_{k} \times\{k\}
$$

is an inverse subsemigroup of $S$. To see this, consider two non-zero elements $x, y$ of this set. Then $x y=0$ unless $x=\left(j, p_{j}^{-1} a p_{k}, k\right), y=\left(k, p_{k}^{-1} b p_{l}, l\right)$ for some $C$ in $M / \rho$, some $j, k, l \in C$ and some $a, b \in H^{C}$. In this case, $x y=\left(j, p_{j}^{-1} a b p_{l}, l\right)$, and so $x y \in V$. Also,

$$
x^{-1}=\left(k, p_{k}^{-1} a^{-1} p_{j}, j\right) \in V .
$$

Thus, $V$ is an inverse subsemigroup of $S$. We have described all the inverse subsemigroups of $S$.

Remark 4.3. If in (4.1) $M=\emptyset$, then $V=\{0\}$. If $M=[n], \rho=[n] \times[n]$ and $H^{[n]}=G$, then $V=S$.

From (4.1) we have

$$
|V \backslash 0|=\sum_{C \in M / \rho}|C|^{2}\left|H^{C}\right| .
$$

Suppose that $\rho$ has classes $C_{1}, C_{2}, \ldots, C_{r}$ containing $m_{1}, m_{2}, \ldots, m_{r}$ elements, respectively, and let us write $H^{C_{i}}$ as $H_{i}$. Then

$$
|V \backslash 0|=\sum_{i=1}^{r} m_{i}^{2}\left|H_{i}\right|
$$

Lemma 4.4. Let $m=m_{1}+m_{2}+\cdots+m_{r}$, with $m_{i} \geqslant 1$ for all $i$. Then

(1) if $r \geqslant 1$, then $m^{2} \geqslant m_{1}^{2}+\cdots+m_{r}^{2}$;

(2) if $r \geqslant 2$, then $(m-1)^{2}+1 \geqslant m_{1}^{2}+\cdots+m_{r}^{2}$.

Proof. Part (1) is a standard inequality. As for part (2), notice that $1 \leqslant m_{r} \leqslant m-1$ and that

$$
\left(m-m_{r}\right)^{2} \geqslant m_{1}^{2}+\cdots+m_{r-1}^{2}
$$

Hence,

$$
\begin{aligned}
{\left[(m-1)^{2}+1\right]-\left[m_{1}^{2}+\cdots+m_{r}^{2}\right] } & \geqslant\left[(m-1)^{2}+1\right]-\left[\left(m-m_{r}\right)^{2}+m_{r}^{2}\right] \\
& =2\left(m_{r}-1\right)\left[(m-1)-m_{r}\right] \geqslant 0,
\end{aligned}
$$

as required. Looking at (4.2), suppose first that $|M|=m \leqslant n-1$. Then

$$
|V \backslash 0|=\sum_{i=1}^{r} m_{i}^{2}\left|H_{i}\right| \leqslant|G| \sum_{i=1}^{r} m_{i}^{2} \leqslant|G| m^{2} \leqslant|G|(n-1)^{2} .
$$


Next, suppose that $M=[n]$, and that $\rho=[n] \times[n]$, the universal relation. Then we have only one equivalence class and $r=1, m_{1}=n$ in (4.2). So $|V \backslash 0|=n^{2}|H|$, where $H$ is a subgroup of $G$. If $H=G$, then $V=S$. If $H$ is a proper subgroup of $G$, then $|H| \leqslant \frac{1}{2}|G|$, and so

$$
|V \backslash 0| \leqslant \frac{1}{2}|G| n^{2} .
$$

Finally, suppose that $M=[n]$ and that $\rho$ has at least two classes. Suppose, in fact, that there are $r \rho$-classes $C_{1}, C_{2}, \ldots, C_{r}$, where $\left|C_{i}\right|=m_{i}(i=1, \ldots, r)$ and $m_{1}+m_{2}+\cdots+$ $m_{r}=n$, then

$$
\begin{aligned}
|V \backslash 0| & =\sum_{i=1}^{r} m_{i}^{2}\left|H_{i}\right| \leqslant|G| \sum_{i=1}^{r} m_{i}^{2} \leqslant|G|\left[(m-1)^{2}+1\right] \\
& =|G|\left[(n-1)^{2}+1\right]=|G|\left(n^{2}-2 n+2\right) .
\end{aligned}
$$

From (4.3), (4.4) and (4.5) we see that if $V$ is a proper inverse subsemigroup of $S$ then

$$
|V| \leqslant\left(n^{2}-2 n+2\right)|G|+1 .
$$

If we take $M=[n]$, the equivalence $\rho$ with two classes, $\{1, \ldots, n-1\}$ and $\{n\}$, and $H^{\{1, \ldots, n-1\}}=H^{\{n\}}=G$, we obtain an inverse subsemigroup

$$
\{0\} \cup\{(i, g, j): i, j=1, \ldots, n-1, g \in G\} \cup\{(n, g, n): g \in G\}
$$

of order $\left(n^{2}-2 n+2\right)|G|+1$, and we now know that this is the best possible.

We deduce that $r_{5}^{I} B(G, n)=\left(n^{2}-2 n+2\right)|G|+2$.

Corollary 4.5. $r_{5}^{I}\left(B_{n}\right)=n^{2}-2 n+4$.

We end the paper by applying Theorem 4.2 to a standard inverse semigroup of permutations. First, however, we consider $r_{5}^{I}\left(\mathcal{I}_{n}\right)$, where $\mathcal{I}_{n}$ is the symmetric inverse semigroup on $X=\{1,2, \ldots, n\}$.

Theorem 4.6. For $n \geqslant 3$,

$$
r_{5}^{I}\left(\mathcal{I}_{n}\right)=\sum_{r=0}^{n}\left(\begin{array}{l}
n \\
r
\end{array}\right)^{2} r !-\frac{1}{2} n !+1 .
$$

Proof. The inverse semigroup $\mathcal{I}_{n}$ has $n+1 \mathcal{J}$-classes, $\mathcal{J}_{0}, \mathcal{J}_{1}, \ldots, \mathcal{J}_{n}$, where $\mathcal{J}_{r}=$ $\left\{\alpha \in \mathcal{I}_{n}:|\operatorname{dom} \alpha|=r(=|\operatorname{im} \alpha|)\right\}$. The $\mathcal{J}$-class $\mathcal{J}_{0}$ consists of the empty map usually denoted by $\{0\}$ and $\mathcal{J}_{n}$ coincides with the symmetric group $S_{n}$.

Consider the proper inverse semigroup of $\mathcal{I}_{n}, V=\mathcal{J}_{0} \cup \mathcal{J}_{1} \cup \cdots \cup \mathcal{A}_{n}$, where $A_{n}$ is the alternating group.

In fact, $V$ is the largest proper inverse subsemigroup of $\mathcal{I}_{n}$. It is clear that it is the largest proper inverse subsemigroup whose intersection with $S_{n}$ is a proper subgroup. So now consider $U$, a proper inverse subsemigroup of $\mathcal{I}_{n}$ such that $S_{n} \subseteq U$.

Now, if $U \cap \mathcal{J}_{n-1} \neq \emptyset$, then $U=\mathcal{I}_{n}$ (see $[2]$ ). 
So let $U \subseteq \mathcal{J}_{0} \cup \mathcal{J}_{1} \cup \cdots \cup \mathcal{J}_{n-2} \cup S_{n}$. Now

$$
\begin{aligned}
|U| & \leqslant\left|\mathcal{I}_{n}\right|-\left|\mathcal{J}_{n-1}\right|=\left|\mathcal{I}_{n}\right|-n^{2}(n-1) ! \leqslant\left|\mathcal{I}_{n}\right|-\frac{1}{2} n ! \\
& =\sum_{r=0}^{n}\left(\begin{array}{l}
n \\
r
\end{array}\right)^{2} r !-\frac{1}{2} n !=|V| .
\end{aligned}
$$

Consequently,

$$
r_{5}^{I}\left(I_{n}\right)=\sum_{r=0}^{n}\left(\begin{array}{l}
n \\
r
\end{array}\right)^{2} r !-\frac{1}{2} n !+1
$$

as required.

Consider now $S P_{n}=\left\{\alpha \in \mathcal{I}_{n}:|\operatorname{dom} \alpha|<n\right\}$, the inverse semigroup of proper subpermutations of $X=\{1,2, \ldots, n\}$, where $n \geqslant 3$. The inverse semigroup $S P_{n}$ has $n$ $\mathcal{J}$-classes. The top $\mathcal{J}$-class in $S P_{n}$ is $\mathcal{J}_{n-1}$, and the associated principal factor $P_{n-1}=$ $S P_{n} /\left(\mathcal{J}_{0} \cup \mathcal{J}_{1} \cdots \cup \mathcal{J}_{n-2}\right)$ may be thought of in the usual way as $\mathcal{J}_{n-1} \cup\{0\}$, where the product in $P_{n-1}$ of two elements of $\mathcal{J}_{n-1}$ is the product in $S P_{n}$ if it lies in $\mathcal{J}_{n-1}$ and is 0 otherwise.

Since the principal factor $P_{n-1}$ is a Brandt semigroup $B(G, n)$, where $G=S_{n-1}$ (see $[2,6]$ ), the symmetric group on $\{1, \ldots, n-1\}$ and $I=\{1,2, \ldots, n\}$, we have, as a consequence of Theorem 4.2, an inverse subsemigroup $S \cup \mathcal{J}_{n-2} \cup \cdots \cup \mathcal{J}_{0}$, where $S$ is of order $(n-1) !\left(n^{2}-2 n+2\right)$. This is certainly the largest proper inverse subsemigroup not containing $J_{n-1}$, and, indeed, is the largest proper inverse subsemigroup of $S P_{n}$, for it is well known that $\left\langle\left\langle J_{n-1}\right\rangle\right\rangle=S P_{n}$ (see [1]). Hence we obtain the following theorem.

Theorem 4.7. Let $S P_{n}=\left\{\alpha \in I_{n}:|\operatorname{dom} \alpha|<n\right\}$ be the inverse semigroup of proper subpermutations of $X=\{1,2, \ldots, n\}$. Then, if $n \geqslant 3$,

$$
r_{5}^{I}\left(S P_{n}\right)=\sum_{r=0}^{n-2}\left(\begin{array}{l}
n \\
r
\end{array}\right)^{2} r !+(n-1) !\left(n^{2}-2 n+2\right)+1
$$

Acknowledgements. The author acknowledges, with thanks, the support of the Fundação para a Ciência e a Tecnologia, the Fundacão Calouste Gulbenkian and Projecto Praxix XXI. The author also acknowledges the assistance of Professor John M. Howie in preparing this material for publication.

\section{References}

1. G. M. S. Gomes AND J. M. HowIE, On the ranks of certain finite semigroups of transformations, Math. Proc. Camb. Phil. Soc. 101 (1987), 395-403.

2. J. M. HowIE, Fundamentals of semigroup theory (Oxford University Press, 1995).

3. J. M. Howie AND M. I. MARques Ribeiro, Rank properties in finite semigroups, Commun. Algebra 27 (1999), 5333-5347.

4. J. M. Howie AND M. I. MARQUes Ribeiro, Rank properties in finite semigroups, II, The small rank and the large rank, S. E. Asian Bull. Math. 24 (2000), 231-237. 
5. E. MARCZEWSKI, Independence in abstract algebras: results and problems, Colloq. Math. 14 (1966), 169-188.

6. M. PETRICH, Inverse semigroups (Wiley, 1984). 